PROCEEDINGS OF THE AMERICAN MATHEMATICAL SOCIETY

Volume 124, Number 9, September 1996

\title{
LOCALLY INJECTIVE MAPS IN O-MINIMAL STRUCTURES WITHOUT POLES ARE SURJECTIVE
}

\author{
ADAM H. LEWENBERG
}

(Communicated by Andreas R. Blass)

\begin{abstract}
If $f: \mathbf{R}^{m} \rightarrow \mathbf{R}^{m}$ is continuous and locally injective, then $f$ is in fact surjective and a homeomorphism, provided $f$ is definable in an o-minimal expansion without poles of the ordered additive group of real numbers; 'without poles' means that every one-variable definable function is locally bounded. Some general properties of definable maps in o-minimal expansions of ordered abelian groups without poles are also established.
\end{abstract}

\section{INTRODUCTION}

Let $\mathcal{R}$ be an expansion of a (nontrivial) divisible ordered abelian group; note that the ordering on $\mathcal{R}$ is dense. The topology on $\mathcal{R}$ is the interval topology which has the open intervals as a basis; the topology on $\mathcal{R}^{n}$ is the inherited product topology. A subset $S$ of $\mathcal{R}^{n}$ is bounded if there is some positive $r \in \mathcal{R}$ such that $S \subseteq[-r, r]^{n}$. The structure $\mathcal{R}$ is o-minimal if every (first-order) definable subset of $\mathcal{R}$ is the finite union of points and open intervals (here the language used for defining sets extends $\{<, 0,+,-\}$, the language of ordered abelian groups). Throughout, "definable" will mean definable with parameters from the structure; to indicate definable without parameters we will write "0-definable".

We say that the structure $\mathcal{R}$ has no poles if every definable function $f: \mathcal{R} \rightarrow \mathcal{R}$ is locally bounded, that is, each point of $\mathcal{R}$ has a neighborhood on which $f$ is bounded.

The main result proved in this paper is the following.

Theorem. Let $\mathcal{R}$ be an o-minimal expansion of $(\mathbf{R},<, 0,+,-)$ without poles. Let $f: \mathbf{R}^{m} \rightarrow \mathbf{R}^{m}$ be definable in $\mathcal{R}$. If $f$ is continuous and locally injective, then $f$ is a homeomorphism onto $\mathbf{R}^{m}$.

This result extends a known result for piecewise linear maps; see [ER]. The main source of structures without poles is the following result from Theorem 1.2 of [Pe] or [PSS].

Proposition. Let $B_{i} \subseteq \mathbf{R}^{n_{i}}$ be bounded, $i \in I$. The structure

$$
\mathcal{R}=\left(\mathbf{R},<, 0,+,-,\left\{\lambda_{a}\right\}_{a \in \mathbf{R}},\left\{B_{i}\right\}_{i \in I}\right)
$$

Received by the editors May 27, 1994 and, in revised form, March 14, 1995.

1991 Mathematics Subject Classification. Primary 03C60, 06F20; Secondary 26B99, 54C30.

Key words and phrases. Piecewise linear topology, PL-topology, o-minimal theory, o-minimal structure, proper map, surjective local homeomorphism.

(C)1996 American Mathematical Society 
(where $\lambda_{a}$ is scalar multiplication by a) has no poles. (Note that if in addition all the $B_{i}$ are definable in a common o-minimal expansion of the additive group of real numbers, then $\mathcal{R}$ is o-minimal.)

\section{Notation AND General FACTS}

Let $\mathcal{L}$ be the language of $\mathcal{R}$. A fundamental fact from the theory of o-minimal structures is that if $\mathcal{R}$ is o-minimal, then every $\mathcal{L}$-structure elementarily equivalent to $\mathcal{R}$ is also o-minimal. Furthermore, if $\mathcal{R}$ is o-minimal and some nonzero element of $\mathcal{R}$ is 0 -definable, then $\operatorname{Th}(\mathcal{R})$, the complete theory of $\mathcal{R}$, has definable skolem functions. Having definable skolem functions implies that $\left\{t^{\mathcal{R}}: t\right.$ is a variablefree $\mathcal{L}$-term $\}$ is the underlying set of an elementary substructure $\mathcal{P} \preceq \mathcal{R}$, and $\mathcal{P}$ is a prime model of $\operatorname{Th}(\mathcal{R})$. In light of this, from now on we assume that some nonzero element of $\mathcal{R}$ is 0 -definable. If this were not the case, then we could choose some nonzero element of $\mathcal{R}$ and add a constant symbol for it to the language - this would not change the class of definable sets (although it would change the class of 0 -definable sets).

If $f: \mathcal{R} \rightarrow \mathcal{R}$ is definable and locally bounded, then we say that $f$ has no poles in $\mathcal{R}$. Assume that $\mathcal{R}$ is o-minimal and that every 0 -definable function from $\mathcal{R}$ to $\mathcal{R}$ has no poles in $\mathcal{R}$. We will show that then every definable function from $\mathcal{R}$ to $\mathcal{R}$ has no poles in $\mathcal{R}$ either. To see this, assume that $f: \mathcal{R} \rightarrow \mathcal{R}$ is a definable function with a pole in $\mathcal{R}$ and let $\varphi\left(r_{1}, \ldots, r_{n}, x, y\right)$ be the formula defining $f$ where $r_{1}, \ldots, r_{n} \in \mathcal{R}$. So, $\operatorname{Th}(\mathcal{R})$ proves the sentence " $\exists z_{1}, \ldots, z_{n}\left[\varphi\left(z_{1}, \ldots, z_{n}, x, y\right)\right.$ defines a total function and has a pole]" (which has no parameters) and so there are $p_{1}, \ldots, p_{n}$ in the prime model $\mathcal{P}$ such that $\varphi\left(p_{1}, \ldots, p_{n}, x, y\right)$ is 0 -definable and defines a function $g: \mathcal{P} \rightarrow \mathcal{P}$ which has a pole. But $\mathcal{P} \preceq \mathcal{R}$ and thus $g^{\mathcal{R}}: \mathcal{R} \rightarrow \mathcal{R}$ is 0-definable with a pole, contradicting our assumption on $\mathcal{R}$.

Thus "having no poles" in $\mathcal{R}$ is preserved under elementary equivalence: if $\mathcal{R}$ is o-minimal and has no poles and $\mathcal{R}^{\prime} \equiv \mathcal{R}$, then $\mathcal{R}^{\prime}$ has no poles.

Notation. We define $\pi_{n}: \mathcal{R}^{n} \rightarrow \mathcal{R}^{n-1}$ by $\pi_{n}\left(x_{1}, \ldots, x_{n-1}, x_{n}\right)=\left(x_{1}, \ldots, x_{n-1}\right)$ for $n>1$. If $S \subseteq \mathcal{R}^{m+n}$ and $x \in \mathcal{R}^{m}$, define $S_{x}:=\left\{y \in \mathcal{R}^{n}:(x, y) \in S\right\}$, the fiber of $S$ over $x$.

\section{REsults}

From now on $\mathcal{R}$ is assumed to be o-minimal without poles.

Proposition. Let $S \subseteq \mathcal{R}^{n}$ be a bounded definable set, and let $f: S \rightarrow \mathcal{R}$ be a definable function. Then $f(S)$ is bounded.

Proof. By induction on $n$. Let $n=1$. Use the Monotonicity Theorem (see [KPS]) to decompose $S$ into a finite number of subintervals so that $f$ is monotone on each subinterval. Since each subinterval has its endpoints in $\mathcal{R}$ and is bounded, and there can be no functions with poles, $f$ must be bounded on each subinterval and hence must be bounded on all of $S$.

Suppose $n>1$. If $a \in \pi_{n} S$, then $S_{a}$ is a bounded set, and so by induction the function $x \mapsto f(a, x): S_{a} \rightarrow \mathcal{R}$ is bounded. Thus it makes sense to define a function $h: \pi_{n} S \rightarrow \mathcal{R}$ by $h(a):=\sup _{x \in S_{a}}|f(a, x)|$ (since in an o-minimal structure $\mathcal{R}$ every definable bounded subset of $\mathcal{R}$ has a supremum in $\mathcal{R}$ ). Note that if $h$ is bounded on $\pi_{n} S$, then $f$ must be bounded on $S$. But $\pi_{n} S$ is a bounded definable subset of $\mathcal{R}^{n-1}$ and so by the inductive hypothesis, $h$ is bounded on $\pi_{n} S$. 
Definition. Let $X \subseteq \mathcal{R}^{n}$ be a definable set. A definable curve in $X$ is a definable map $\gamma:(a, b) \rightarrow X$ where $(a, b) \subseteq \mathcal{R}$ and $-\infty<a<b<+\infty$. If $\gamma:(a, b) \rightarrow X$ is a definable curve in $X$ and $\lim _{t \rightarrow b^{-}} \gamma(t)=p \in \mathcal{R}^{n}$, we write $\gamma \rightarrow p$. If there exists a (necessarily unique) point $p \in \mathcal{R}^{n}$ such that $\gamma \rightarrow p$ we say that $\gamma$ is completeable; if in addition $p \in X$, we say that $\gamma$ is completeable in $X$.

We can now use the above proposition and Curve Selection to prove the following facts. (For a statement and proof of Curve Selection, see [vdDL].)

Facts. Let $X \subseteq \mathcal{R}^{n}$ be definable. (1) Every definable curve in $X$ is completable. (2) $X$ is closed if and only if every definable curve in $X$ is completeable in $X$. (3) Let $Y \subseteq \mathcal{R}^{m}$ be definable. If $f: X \rightarrow Y$ is a definable map with $p \in X$, then $f$ is continuous at the point $p$ if and only if for each definable curve $\gamma$ in $X$ with $\gamma \rightarrow p$ we have $f(\gamma) \rightarrow f(p)$.

Proposition. Let $S \subseteq \mathcal{R}^{m}$ be definable and $f: S \rightarrow \mathcal{R}^{n}$ a definable map. The graph of $f$ is closed in $S \times \mathcal{R}^{n}$ if and only if $f$ is continuous.

Proof. That the continuity of $f$ implies that the graph of $f$ is closed in $S \times \mathcal{R}^{n}$ is well known. On the other hand, assume that the graph of $f$ is closed in $S \times \mathcal{R}^{n}$. Let $p \in S$. It suffices by (3) above to show that for every definable curve $\gamma$ in $S$ if $\gamma \rightarrow p$, then $f(\gamma) \rightarrow f(p)$. Fix a definable curve $\gamma:(a, b) \rightarrow S$ such that $\lim _{t \rightarrow b^{-}} \gamma(t)=p$. We need to show that $f(\gamma) \rightarrow f(p)$. Let $X$ denote the graph of $f$. Define in $X$ a definable curve $\gamma_{X}$ by $t \mapsto(\gamma(t), f(\gamma(t))):(a, b) \rightarrow X$. Since $X$ is closed, $\gamma_{X}$ is completeable, say $\gamma_{X} \rightarrow(z, f(z)) \in X$ where $z \in S$. It is easy to see that $\gamma \rightarrow z$ and $f(\gamma) \rightarrow f(z)$ and hence $z=p$, which shows that $f(\gamma) \rightarrow f(p)$ as desired.

Proposition. Let $S$ be a definable closed subset of $\mathcal{R}^{m}$ and $f: S \rightarrow \mathcal{R}^{n}$ a definable continuous map. Then $f(S)$ is closed.

Proof. We will show that every definable curve in $f(S)$ is completeable in $f(S)$, which implies $f(S)$ is closed. Let $\gamma:(a, b) \rightarrow f(S)$ be a definable curve such that $\gamma \rightarrow z \in \mathcal{R}^{n}$. It suffices to show that $z \in f(S)$. Let $\Gamma \subseteq f(S)$ be the image of $(a, b)$ under $\gamma$. By Definable Choice (see [vdDL]) there is a definable set $B \subseteq S$ such that $\left.f\right|_{B}$ is injective and $f(B)=\Gamma$; let $g: \Gamma \rightarrow B$ be inverse to $f$. Define a new definable curve $\eta:(a, b) \rightarrow B$ by $\eta(t):=g(\gamma(t))$. As $S$ is closed, the definable curve $\eta$ is completeable in $S$, say $\eta \rightarrow s \in S$. Now,

$$
s=\lim _{t \rightarrow b^{-}} \eta(t)
$$

and as $f$ is continuous,

$$
f(s)=f\left(\lim _{t \rightarrow b^{-}} \eta(t)\right)=\lim _{t \rightarrow b^{-}} f(\eta(t))=\lim _{t \rightarrow b^{-}} f(g(\gamma(t)))=\lim _{t \rightarrow b^{-}} \gamma(t)=z .
$$

Thus, $z \in f(S)$.

Theorem. Let $\mathcal{R}$ be an o-minimal expansion of $(\mathbf{R},<, 0,+,-)$ without poles. Let $f: \mathbf{R}^{m} \rightarrow \mathbf{R}^{m}$ be definable in $\mathcal{R}$. If $f$ is continuous and locally injective, then $f$ is a homeomorphism onto $\mathbf{R}^{m}$.

Proof. Assume $f$ is continuous and locally injective. By the previous proposition, $f\left(\mathbf{R}^{m}\right)$ is closed in $\mathbf{R}^{m}$. It follows from Invariance of Domain that the image $f\left(\mathbf{R}^{m}\right)$ is open in $\mathbf{R}^{m}$ and that $f$ is a local homeomorphism (see [D]). Thus, $f\left(\mathbf{R}^{m}\right)$ is open 
and closed in $\mathbf{R}^{m}$ and so $f\left(\mathbf{R}^{m}\right)$ is equal to all of $\mathbf{R}^{m}$. Therefore $f$ is surjective and a local homeomorphism.

A continuous map $g: X \rightarrow Y$ where $X$ and $Y$ are both locally compact and Hausdorff is called proper if the inverse image of every compact set in $Y$ is compact in $X$. As $\mathcal{R}$ is o-minimal, for each $x \in \mathcal{R}$ the fiber $f^{-1}(x)$ is finite. Together with the fact that $f$ is closed and continuous, this shows that $f$ is a proper map (see Theorem I in $\$ 10.2$, Chapter I of [B]). But a surjective proper local homeomorphism is a covering map (see $[\mathrm{F}]$ ). As $\mathbf{R}^{m}$ is simply connected, $f$ must in fact be a homeomorphism.

Remark. The above proof goes through when we replace $\mathbf{R}^{m}$ by any closed, simply connected definable subset of $\mathbf{R}^{m}$.

Remark. The role of surjectivity in the proof of the above is essential. So perhaps all we need for $f: \mathbf{R}^{n} \rightarrow \mathbf{R}^{n}$ to be a homeomorphism is that it be merely surjective and locally injective, and not necessarily definable in an o-minimal expansion without poles of $(\mathbf{R},<, 0,+,-)$. However, as the following example shows, this is not the case.

Let $X:=\{(x, y): x>0\}$, the right open half-plane in $\mathbf{R}^{2}$, and let $Y:=$ $\{(x, y): x>0$ and $y \geq 0\}$. Let $g$ be the function $(x, y) \mapsto\left(e^{x}, y\right): \mathbf{R}^{2} \rightarrow X$, a homeomorphism. Define the function $h: X \rightarrow \mathbf{R}^{2}-\{(0,0)\}$ so that $h$ is the identity on $X-Y=$ the open lower right-hand quadrant; if $p \in Y$, then $p=r e^{2 \pi i \theta}$ where $r>0$ and $0 \leq \theta<1 / 4$ and in this case define $h(p):=r e^{8 \pi i \theta}$ (here we identify the points of $\mathbf{R}^{2}$ with the points of $\mathbf{C}$, the complex plane). The function $h$ is a surjective local homeomorphism from $X$ onto $\mathbf{R}^{2}-\{(0,0)\}$. We now define a new map $H:\{(x, y): 0<x$ and $y \leq-1\} \rightarrow \mathbf{R}^{2}-\{(0,-1)\}$ in the same way as we defined $h$, namely an exponential map wrapping the open quadrant around the point $(0,-1)$ (see Figure 1).

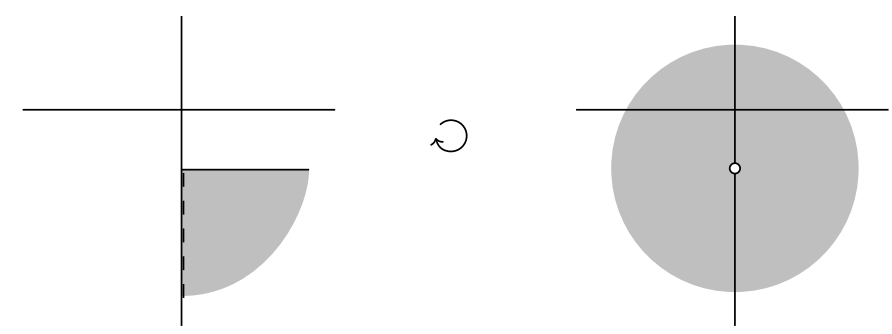

Figure 1

Let $f$ be the pasting together of $h$ and $H$, that is, let $f: X \rightarrow \mathbf{R}^{2}$ where $f$ agrees with $h$ except on the domain of $H$ where it agrees with $H$. By construction, $f$ is a local homeomorphism. Let $f^{\prime}:=f \circ g$. Note that $f^{\prime}$ is a surjective local homeomorphism from $\mathbf{R}^{2}$ onto $\mathbf{R}^{2}$. In particular, $f^{\prime}$ is not a homeomorphism, as a small neighborhood of the origin lifts to something like that shown in Figure 2.

This example is due to M. E. Hamstrom. In fact, $f^{\prime}$ is not even a generalized covering map as the upper shaded region (in Figure 2) is not mapped homeomorphically onto its image (see $[\mathrm{Hu}]$ for the definition of generalized covering); this contradicts exercise $Q$ on page 105 of $[\mathrm{Hu}]$. 


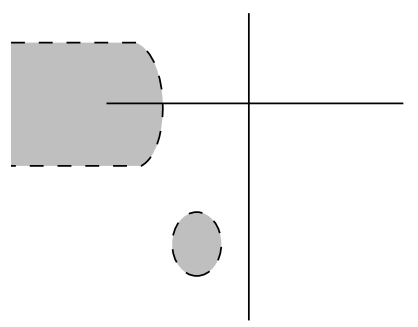

FiguRE 2

This example is definable in the o-minimal structure $\left(\mathbf{R},+, \cdot, \exp ,\left.\sin \right|_{[0, \pi]}\right)$. Of course, this example is not definable in an o-minimal expansion without poles of $(\mathbf{R},<, 0,+,-)$. Note that the role the exponential function played can be filled by semialgebraic (though nonsmooth) functions. Thus, another (but similar) example of a noninjective surjective local homeomorphism from $\mathbf{R}^{2}$ to $\mathbf{R}^{2}$ is definable in $(\mathbf{R},<, 0,+, \cdot)$.

Added in proof. The proof of the above theorem uses some very special properties of Euclidean space, for example, Invariance of Domain and the fact that Euclidean space has no nontrivial covering spaces. However, Alex Wilkie has pointed out a very nice generalization eliminating the reliance on special properties of the real numbers at the cost of a small strengthening of the hypothesis. We make this explicit in the following theorem.

Theorem. Let $\mathcal{R}=(R,<, 0,1,+,-, \ldots)$ be an o-minimal expansion of an ordered abelian group with a 0-definable nonzero element without poles. Let $f: R^{m} \rightarrow R^{m}$ be definable in $\mathcal{R}$. If $f$ is continuous and a local homeomorphism, then $f$ is a homeomorphism onto $R^{m}$.

Proof. By the third proposition of this section, $f\left(R^{m}\right)$ is closed in $\mathcal{R}^{m}$. As $f$ is a local homeomorphism, in particular $f$ is an open map, and so $f\left(R^{m}\right)$ is also open in $R^{m}$. Thus, $f\left(R^{m}\right)$ is a definable subset of $R^{m}$ which is both closed and open. But $\mathcal{R}^{m}$ is definably connected, which implies that $f\left(R^{m}\right)$ must be all of $R^{m}$. Hence, $f$ is a surjective local homeomorphism.

For each natural number $n$ define $S_{n}$ to be all those elements $y$ of $R^{m}$ such that $\left|f^{-1}(y)\right|=n$. Since $f$ is locally injective, the fiber $f^{-1}(y)$ is discrete for each $y \in R^{m}$, and as $\mathcal{R}$ is o-minimal, $f^{-1}(y)$ must in fact be finite. In addition, as $f$ is surjective, we can conclude that $R^{m}=S_{1} \cup S_{2} \cup \cdots$. By the Finiteness Lemma (see $[\mathrm{vdD}]$ ) we can further conclude that there is some largest $N$ such that $S_{N}$ is nonempty.

We next claim that $S_{N}$ is both open and closed. That $S_{N}$ is open is straightforward and is left to the reader. We will prove that $S_{N}$ is closed. Towards a contradiction assume that $S_{N}$ is not closed, witnessed by $p \notin S_{N}$, an accumulation point of $S_{N}$. Let $f^{-1}(p)=\left\{q_{1}, \ldots, q_{n}\right\}$ where $0<n<N$. By an easy argument, we can assume that there are disjoint open neighborhoods $U_{1}, \ldots, U_{n}$ of $q_{1}, \ldots, q_{n}$, and a neighborhood $V$ of $p$ such that $f$ maps each $U_{i}$ homeomorphically onto $V$ via $f$. Let $\gamma:[0, \varepsilon) \rightarrow S_{N} \cap V$ be an injective continuous definable path in $S_{N} \cap V$ such that $\gamma \rightarrow p$. Let $\Gamma$ be the image of $[0, \varepsilon)$ under $\gamma$, and let $X:=f^{-1}(\Gamma \cup\{p\})$. Furthermore, let $U_{i}^{\prime}:=X \cap U_{i}$ and $W:=X-\left(U_{1}^{\prime} \cup \cdots \cup U_{n}^{\prime}\right)$. Note that because $\Gamma \subseteq S_{N}$, it must be that $f(W)=\Gamma$. Because $\Gamma \cup\{p\}$ is closed, $X$ is also closed. 
The $U_{i}^{\prime}$ are all open in $X$, and so their union is open in $X$. Thus, $W$ is closed in $X$ and hence closed in $R^{m}$. So, $f(W)$ is closed. But $f(W)=\Gamma$, a nonclosed set. Contradiction.

Thus, $S_{N}$ is a nonempty definable open and closed subset of $R^{m}$, and hence $S_{N}=R^{m}$. So, $f: R^{m} \rightarrow R^{m}$ is an $N$-to-1 definable surjective map. If $S \subseteq R^{m}$ is definable, we let $E(S) \in \mathbf{Z}$ denote the Euler characteristic of $S$, a useful definable invariant. In particular, the Euler characteristic of a definable set is preserved under a definable injective map; for more details on Euler characteristic, see [vdD]. Two other useful facts about Euler characteristic are that $E\left(R^{m}\right)= \pm 1$ and that if $f: A \rightarrow B$ is definable and $k$-to-1, then $E(A)=k E(B)$. These two facts imply that $N$ must be 1 . So $f$ must in fact be a homeomorphism.

Remark. It is not hard to show that the above proof goes through when we replace $R^{m}$ by any closed, connected definable subset of $R^{m}$ whose Euler characteristic is not 0 .

\section{ACKNOWLEDGMENTS}

I thank Lou van den Dries for the inspiration, M. E. Hamstrom for the example, Arthur Woerheide for suggestions that improved the exposition and Alex Wilkie for improvements to the main result.

\section{REFERENCES}

[B] N. Bourbaki, General topology, Chapters 1-4, Springer-Verlag, Berlin, 1989. MR 90a:54001a

[vdD] Lou van den Dries, O-minimal structures and tame topology, preprint, 1995.

[vdDL] Lou van den Dries and Adam H. Lewenberg, T-convexity and tame extensions, J. Symbolic Logic 60 (1995). MR 96a:03048

[D] Albrecht Dold, Lectures on algebraic topology, second edition, Springer-Verlag, Berlin, 1980. MR 82c: 55001

[ER] B. Curtis Eaves and Uriel G. Rothblum, Dines-Fourier-Motzkin quantifier elimination and an application of corresponding transfer principles over ordered fields, Math. Programming 53 (1992), 307-321. MR 93h:03037

[F] Otto Forster, Lectures on Riemann surfaces, Graduate Texts in Math., vol. 81, SpringerVerlag, New York, 1981, pp. 28-29. MR 83d:30046

[Hu] Sze-Tsen Hu, Homotopy theory, Academic Press, New York, 1959, pp. 104-105. MR 21:5186

[KPS] Julia F. Knight, Anand Pillay, and Charles Steinhorn, Definable sets in ordered structures. II, Trans. Amer. Math. Soc. 295 (1986), 593-605. MR 88b:03050b

[OR] J. M. Ortega and W. C. Rheinboldt, Iterative solution of nonlinear equations in several variables, Academic Press, New York, 1970, pp. 133-138. MR 42:8686

[Pe] Ya'acov Peterzil, A structure theorem for semibounded sets in the reals, J. Symbolic Logic 57 (1992), 779-794. MR 94a:03061

[Pi] Anand Pillay, On groups and fields definable in o-minimal structures, J. Pure Appl. Algebra 53 (1988), 239-255. MR 89i:03069

[PSS] A. Pillay, P. Scowcroft, and C. Steinhorn, Between groups and rings, Rocky Mountain J. Math. 19 (1989), 871-885. MR 91f:03076

[S] Michael Spivak, A comprehensive introduction to differential geometry, Vol. 1, second edition, Publish or Perish, Houston, TX, 1979, p. 3. MR 82g:53003a

Department of Mathematics, University of Illinois at Urbana-Champaign, Urbana, ILLINOIS 61801

E-mail address: adams@math.uiuc.edu 